\title{
NOTES
}

\section{Lithium Bis(trimethylsilyl)amide Induced Anionic Polymerization of Methyl Methacrylate}

\author{
Yukio NagASaKI, ${ }^{* \dagger}$ Hiroaki NishizuKa, ${ }^{* *}$ and Teiji Tsuruta ${ }^{* *}$ \\ * Department of Materials Science and Technology, \\ Science University of Tokyo, Noda 278, Japan \\ ** Department of Industrial Chemistry, Science University of Tokyo, \\ Shinjuku-ku, Tokyo 162, Japan
}

(Received March 8, 1995)

\begin{abstract}
KEY WORDS Anionic Polymerization / Methyl Methacrylate / Lithium Bis(trimethylsilyl)amide / Telechelic Oligomer / prim-Amino-ended Poly(methyl methacrylate) /
\end{abstract}

Lithium-nitrogen-bonded compounds (lithium alkylamides) are known to induce the anionic polymerization of vinyl monomers such as butadiene, ${ }^{1}$ isoprene, ${ }^{2}$ styrene, ${ }^{3}$ and divinylbenzene. ${ }^{4}$ This is one way to introduce an amino group at the end of a polymer chain. If lithium alkylamide can be used as an initiator for the anionic polymerization of $\alpha, \beta$-unsaturated esters such as methyl methacrylate (MMA) and methyl acrylate (MA), a new synthetic route for amino-ended poly[(meth)acrylate]s can be created.

Since the basicity of lithium alkylamide having alkyl substituents such as diisopropylamide (LDA) is too strong, it often induces a carbonyl attack toward such compounds and causes some side reactions. ${ }^{5}$ Lithium bis(trimethylsilyl)amide (LBA) is well known as one of the suitable lithiation agents for $\alpha$-carbonyl protons without carbonyl attack. ${ }^{6}$ If LBA is

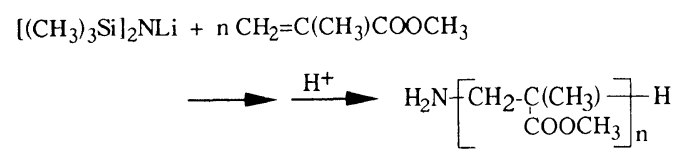

Scheme 1.

\footnotetext{
† To whom correspondence should be addressed.
}

used as the initiator for MMA polymerization, a new route for the synthesis of prim-aminoended PMMA will be available after the hydrolysis of the $\mathrm{N}-\mathrm{Si}$ bond at the end of the polymer chain. This paper reports the anionic polymerization of MMA initiated with LBA.

\section{EXPERIMENTAL}

All experiments for purification and polymerization processes were carried out under purified nitrogen in order to preclude any atmospheric oxygen and moisture.

\section{Materials}

Commercial tetrahydrofuran (THF), toluene (Tol), benzene (Bz), MMA, and hexamethyldisilazane (HMDS) were purified by conventional methods. ${ }^{7}$ Commercial methanol was used as received for the polymer precipitations. A hexane solution of butyllithium (BuLi, Sankyo Chemical Co., Ltd.; $1.6 \mathrm{moll}^{-1}$ ) was used after a double titration. ${ }^{8}$

\section{Polymerization Procedure}

One representative procedure for the anionic 
Table 1. Anionic polymerizations of MMA initiated with LBA

\begin{tabular}{|c|c|c|c|c|c|c|c|c|c|c|}
\hline \multirow{2}{*}{ Run } & \multirow{2}{*}{ Solv. } & \multirow{2}{*}{$\frac{\text { Temp }}{{ }^{\circ} \mathrm{C}}$} & \multirow{2}{*}{$\frac{\text { Time }}{\mathrm{h}}$} & {$[\mathrm{MMA}]_{0}$} & {$[\mathrm{LBA}]_{0}$} & {$[\mathrm{HMDS}]_{0}$} & \multirow{2}{*}{ Conv. $^{\mathrm{a}}$} & \multirow{2}{*}{$\frac{\text { Yield }^{\mathrm{b}}}{\%}$} & \multicolumn{2}{|c|}{$10^{-3} \times M_{n}$} \\
\hline & & & & & $\times 10^{2} \mathrm{moll}^{-}$ & & & & Calcd $^{\mathrm{c}}$ & $\mathrm{GPC}^{\mathrm{d}}$ \\
\hline 1 & THF & 20 & 24 & 23.3 & 3.3 & 0 & - & 0 & - & - \\
\hline 2 & $\mathrm{Bz}$ & 20 & 24 & 23.3 & 3.3 & 0 & - & 46.1 & 0.48 & 3.3 \\
\hline 3 & $\mathrm{Bz}$ & 20 & 24 & 23.3 & 3.3 & 3.3 & - & 13.5 & 0.26 & 3.2 \\
\hline 4 & Tol & -20 & 1 & 23.3 & 3.3 & 0 & 100 & 86.5 & 0.86 & 13.0 \\
\hline
\end{tabular}

${ }^{a}$ Unreacted MMA determined by GC. ${ }^{b}$ Methanol precipitated part. ${ }^{c}$ Calculated by the equation, $M_{n}($ Calcd $)=$ $161+[\mathrm{MMA}]_{0} \cdot$ yield $/[\mathrm{LBA}]_{0} .{ }^{\mathrm{d}}$ Determined by GPC.

polymerizations of MMA is described. In a $100 \mathrm{ml}$ round bottomed-flask equipped with three way stopcock, Bz, HMDS, and BuLi were added via a syringe. After the formation of LBA, MMA was added and the mixture was allowed to react for a certain period. The reaction mixture was then poured into a large amount of methanol ( $c a .20$ times) to precipitate the polymer. The polymer thus obtained was further purified by three reprecipitations from a THF solution to methanol. Finally, a white powder was obtained by freeze-drying with benzene. The detailed reaction conditions and results are summarized in Table I.

\section{Hydrolysis of Silazane at the End of PMMA Chain}

The THF solution of the polymer was poured dropwise into $0.1 \mathrm{~N} \mathrm{HCl}$. The precipitated polymer was dissolved in THF and purified in the same way as previously stated.

\section{Measurements}

Gel permeation chromatography (GPC) was done using a Nihon Seimitsu Kagaku NSLC-200 (TSK-Gel G4000H8 + G3000H8 + G2500H8; Eluent: tetrahydrofuran (THF) $\left.\left(1 \mathrm{ml} \mathrm{min}^{-1}\right)\right)$. Gas chromatography (GC) was done using a Hitachi 663-30 with a PEG-20M ( $2 \mathrm{~m})$ packed column. For NMR measurements, a JEOL FX-100 was used. $10 \mathrm{wt} \%$ of the polymer solution in $\mathrm{CDCl}_{3}$ was employed for the measurement at room temperature.

\section{RESULTS AND DISCUSSION}

Organosilicon compounds are known to stabilize their anions at the $\alpha$-position because of the electron-withdrawing effect. ${ }^{9}$ Consequently, lithium amide with organosilyl substituent(s) shows no carbonyl attack under appropriate conditions. ${ }^{5}$ Such mild reactivity is one of the reasons why LBA has been extensively utilized for the metalation of complicated materials.

When LDA was reacted with MMA in Tol at $-20^{\circ} \mathrm{C}$, no polymerization took place. From GC analysis of the reaction mixture, a new peak appeared in a higher bp area than that of monomer, suggesting carbonyl attack of LDA toward MMA to deactivate the initiator. In contrast to LDA, the mixture of LBA with MMA shows no GC peak in the above bp area using the same conditions. Thus, if LBA has enough nucleophilic reactivity toward the double bond of MMA, it can be utilized as a suitable initiator without carbonyl attack.

The nucleophilic reactivity of LBA toward MMA was examined under several reaction conditions. In THF solution, LBA did not show any reactivity toward MMA. Actually, no polymer was obtained under the condition $[\mathrm{MMA}]_{0} /[\mathrm{LBA}]_{0}=0.233 / 3.3 \times 10^{-2}\left(\mathrm{moll}^{-1}\right)$. THF is reported to coordinate to the lithium cation during anionic polymerization. ${ }^{10}$ Such solvation generally increases the reactivity of the anions. ${ }^{11}$ In the case of MMA polymerization, however, the coordination of a monomer molecule to a lithium cation plays an important role in the reactivity of the initiator. ${ }^{12}$ In apolar 


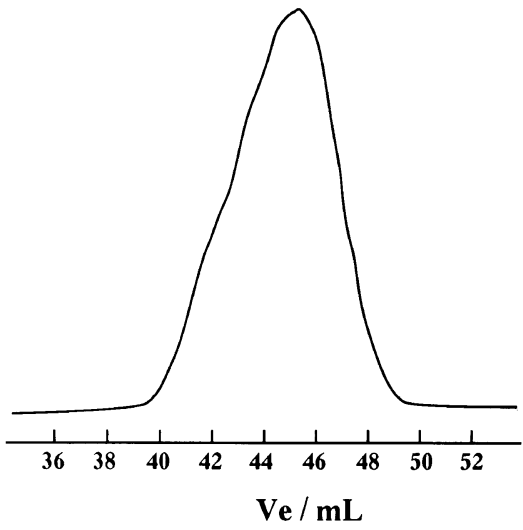

Figure 1. GPC of the poly(methyl methacrylate) obtained by anionic polymerization with lithium bis(trimethylsilyl)amide (LBA) as an initiator (the same sample as in Run 2 in Table I).

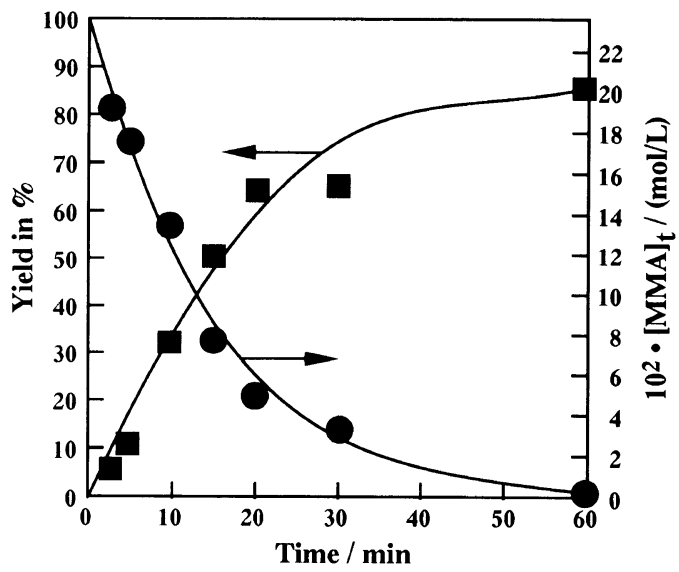

Figure 2. Change in monomer concentration and yields of polymers during the anionic polymerization of MMA initiated with LBA as a function of time (the same conditions as Run 4 in Table I).

solvents such as $\mathrm{Bz}$ and Tol, the coordination of the monomer molecules to the lithium cation is expected to be facilitated because of the absence of THF which solvated to the cation. As shown in Figure 1, the polymer was obtained during the anionic polymerization in $\mathrm{Bz}$ at $20^{\circ} \mathrm{C}$ for $24 \mathrm{~h}$. The molecular weight (MW) of the polymer thus obtained was much higher than that expected by the monomer/ initiator ratio with broad MW distribution (MWD), indicating the low initiation efficiency

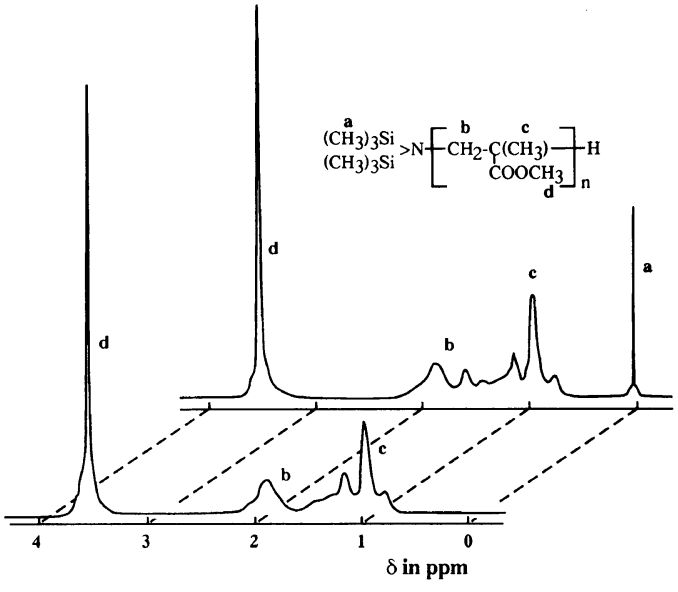

Figure 3. ${ }^{1} \mathrm{H}$ NMR spectrum of the PMMA obtained by LBA induced anionic polymerization before (a) and after (b) acid treatment (the same sample as in Run 2 in Table I).

of LBA due to its reduced reactivity by the electron-withdrawing effect of the $\mathrm{Si}$ atom. In the presence of the free amine, HMDS, only $13 \%$ of the methanol precipitated polymer was obtained (Run 3 in Table I), which suggests that the methanol soluble oligomers might be formed due to chain transfer reactions by HMDS.

It is interesting to note that the polymerization of MMA in Tol at $-20^{\circ} \mathrm{C}$ was almost quantitative only for the 1 -h reaction (Figure 2 and Run 4 in Table I). The molecular weight of the polymer, however, was very high (13000). This can be explained by the higher propagation rate than that at $20^{\circ} \mathrm{C}$ in $\mathrm{Bz}$ due to the higher coordination capacity of the MMA molecule toward the lithium cation at lower temperature though the initiation efficiency was rather low at lower temperatures.

Characterization of the obtained polymer was carried out by ${ }^{1} \mathrm{H}$ NMR spectroscopy. As shown in Figure $3 \mathrm{a}$, the polymer had a trimethylsilyl signal originating from the bis(trimethylsilyl)amino end-group at $0 \mathrm{ppm}$, indicating that the initiation took place by the addition reaction of LBA toward the double bond of the MMA molecule. After the polymer was treated with $0.1 \mathrm{~N} \mathrm{HCl}$, the signal at $0 \mathrm{ppm}$ 
completely disappeared as shown in Figure $3 b$, which indicates that the hydrolysis of the $\mathrm{N}-\mathrm{Si}$ bond proceeded using acid to form the primamino group at the end of the polymer chain. Actually, the polymer after hydrolysis was ninhydrin positive. On the basis of these results, it is concluded that LBA shows an initiation ability toward MMA in apolar solvents. A low polymerization temperature is preferable for smooth polymerization though the initiation efficiency is not very high.

\section{REFERENCES}

1. T. C. Chen, "Anionic Polymerization. VII, Polymerization and Copolymerization with LithiumNitrogen-Bonded Initiator," in "Anionic Polymerization," J. E. MacGrath, Ed., ACS Symposium Series, American Chemical Society, Washington, 1981.

2. N. Yoshino, Y. Yamaki, Y. Nagasaki, and T. Tsuruta, Makromol. Chem., 184, 737 (1983).

3. A. C. Angood, S. A. Hurley, and P. J. T. Tait, $J$.
Poly. Sci., Polym. Chem., Ed., 11, 2777 (1973).

4. a) Y. Nitadori and T. Tsuruta, Makromol. Chem., 179, 2069 (1978).

b) Y. Nagasaki, M. Kato, N. Kato, Y. Mitsuhata, H. Nishizuka, and T. Tsuruta, Bull. Soc. Chim. Bel., 99, 957 (1990).

5. B. J. Wakefield, “Organolithium Methods," Academic Press, London, 1988.

6. B. J. Wakefield, "Organolithium Methods," Academic Press, London, 1988.

7. D. D. Perrin, W. L. F. Armarego, and D. R. Perrin, "Purification of Laboratory Chemicals," 2nd ed., Pergamon Press, Oxford, 1980.

8. H. Gilman and A. H. Haubeim, J. Am. Chem. Soc., 66, 1515 (1944)

9. R. R. Fraser, T. S. Mansour, and S. Savard, Can. J. Chem., 63, 3505 (1985).

10. J. M. Ginn and K. J. Ivin, Makromol. Chem., 139, 47 (1970).

11. T. Shimomura, J. Smid, and M. Szwarc, J. Am. Chem. Soc., 89, 5743 (1967).

12. A. H. Müller, "Kinetics and Mechanisms in the Anionic Polymerization of Methacrylic Esters," in "Recent Advances in Anionic Polymerization," T. E. Hogen-Esch and J. Smid, Ed., Elsevier, New York, N.Y., 1987. 